\title{
Telomere regulation and function during meiosis
}

\author{
Manos Siderakis \& Madalena Tarsounas* \\ Radiation Oncology and Biology, University of Oxford, Churchill Hospital, Oxford, OX3 7LJ, UK; \\ E-mail: madalena.tarsounas@rob.ox.ac.uk \\ * Correspondence
}

Key words: homologous recombination, meiosis, telomerase, telomeres, t-loop

\begin{abstract}
Telomeres are essential for genomic stability and their dysfunction has been implicated in cancer and ageing. The most prominent function of the telomeres is to protect chromosome ends against degradation and fusion, which, in turn, requires maintenance of telomere DNA to a critical length that allows assembly of end-capping structures. During early meiosis, telomeres play the distinctive function of anchoring chromosomes to the inner nuclear membrane. Subsequently, as a consequence of the nuclear membrane polarization, telomeres cluster together into a bouquet configuration, which facilitates pairing and recombination of the homologous chromosomes. Here we review how the two fundamental aspects of telomere maintenance, elongation and protection, contribute to the essential functions performed by telomeres during meiosis.
\end{abstract}

\section{Telomere integrity during meiosis: elongation and capping}

Telomeres are complex structures at chromosome termini, consisting of arrays of repetitive DNA sequences and associated proteins (Blackburn 2001, de Lange 2005). They protect chromosome ends from degradation and fusion, both of which stimulate checkpoint activation, ultimately leading cells to apoptosis or irreversible growth arrest known as replicative senescence (Maser \& DePinho 2002). However, in cells lacking checkpoint responses, short or uncapped telomeres elicit rampant genomic instability, similar to that caused by unrepaired DNA double-strand breaks (DSBs). This type of instability stems from chromosomal fusion-breakage cycles known to generate and amplify chromosomal aberrations, one of the factors promoting the development of cancer. Thus, telomere dysfunction, exhibited either as telomere shortening or loss of telomeric capping structures, is associated with genetic instability and onset of tumorigenesis or premature ageing in mitotically dividing cells.

In contrast with the considerable attention received by somatic telomeres connected with cancer development and progression, as well as organismal ageing, our understanding of the mechanisms of telomere maintenance in meiosis is still at a very early stage. Traditionally, research in this field has focused on the cytological aspects of telomere dynamics in meiosis due to the undoubted aesthetic attraction of the floral arrangement of chromosomes and the dramatic changes in positioning, with relevance to the meiotic chromosome recombination and segregation. However, a complete understanding of meiotic telomeres functions can only be achieved by integrating the cytology with the two features central to telomere maintenance in all types of cells: elongation and protection (capping). Here, we explore the significant contribution of telomere homeostasis and protection to the integrity and function of meiotic chromosomes, ultimately ensuring fertility. The existing evidence 
mainly originates from the meiotic phenotype of the Terc $^{-1-}$ mouse carrying a null mutation in the telomerase RNA component (Blasco et al. 1997, Lee et al. 1998), the only bona fide mouse model for telomere dysfunction generated so far.

\section{Telomere length regulation}

Mammalian telomeres consist of tandem arrays of duplex TTAGGG repeats which vary in length from approximately $10 \mathrm{~kb}$ in humans to $>40 \mathrm{~kb}$ in mouse (de Lange et al. 1990, Zijlmans et al. 1997). The double-stranded telomeric DNA track ends with a 150-nucleotide single-stranded $3^{\prime}$ protrusion of the G-rich strand. This so-called $3^{\prime}$ overhang represents the substrate for telomerase, which provides the most versatile and widely used activity of telomere maintenance (McEachern et al. 2000).

Telomerase is a ribonucleoprotein with reverse transcriptase activity (Greider \& Blackburn 1985, Blasco et al. 1995), which extends the G-rich strand of the telomere, thus preventing gradual loss of DNA sequences from the chromosome ends with each round of replication (Harley et al. 1990, Bodnar et al. 1998). This 'end replication problem' stems from the inability of conventional polymerases to complete synthesis of chromosome ends (Watson 1972). In the absence of telomerase, continuous cell division results in reduction of telomeres to a short critical length, which elicits a checkpoint response similar to the one provoked by DNA damage (Wahl \& Carr 2001, de Lange 2002). For example, in telomerasedeficient mice, telomere attrition leads to end-to-end fusions and cell death (Blasco et al. 1997, Lee et al. 1998, Herrera et al. 1999). Cell growth and telomere homeostasis are restored upon re-introduction of telomerase both in cultured cells and in the Terc $^{-1-}$ mouse (Bodnar et al. 1998, Hemann et al. 2001, Samper et al. 2001). Also, telomerase activation in somatic cells normally lacking detectable levels of this activity is thought to maintain the immortal growth capacity of more than $90 \%$ of human tumours (Hiyama \& Hiyama 2002).

Most mammalian somatic cells, including adult stem cells, do not have sufficient telomerase activity to support telomere replenishment at each cell division, which leads to progressive shortening of telomeres throughout adult life (Harley et al. 1990, Flores et al. 2005). However, certain types of cells in the adult organism retain high telomerase activity essential for their function and viability; a wellstudied example is the germ line (reviewed in Blasco 2005). Consistent with this, in telomerase-negative mice the progressive loss of telomeric sequences leads gradually to infertility, as evidenced by reduced litter size and compromised embryo development (Lee et al. 1998, Herrera et al. 1999), indicating that maintenance of telomere length is essential in the germ line.

Meiosis is an evolutionarily conserved process consisting of two successive divisions leading to generation of haploid cells (gametes). Upon fertilization, these can reconstruct the diploid organism. In mammals, meiosis occurs in testes and ovaries, both consisting of highly heterogeneous cell populations. Telomerase activity was found in fetal, newborn and mature ovaries and testes, but mature oocytes and adult sperm samples lack telomerase activity (Wright et al. 1996).

\section{The ovary}

In females, the oocytes initiate meiosis very early during embryonic development and undergo a prolonged arrest at prophase I, which lasts for weeks in mice and years in humans until puberty. Gonadotrophin stimulation induces resumption of meiosis and progression of competent oocytes through the remaining stages of meiosis I, until metaphase II where they arrest again until fertilization. In the ovary, oocytes are surrounded by somatic cells, which communicate hormone-mediated signals and nourish and protect germ cells.

In contrast to the male germ line, all germ cells in the ovary initiate meiosis within a period of days in the mouse and weeks in humans; therefore, isolation of semi-synchronous oocyte populations has been possible. Measurements of telomerase activity in an in vitro TRAP assay (Kim et al. 1994) have shown a peak in the pre-ovulation oocytes (Eisenhauer et al. 1997) and subsequent decrease in mature oocytes (Wright et al. 2001) and cleavage-stage embryos, until the 8-cell stage. At this point telomerase is reactivated and significant increase in activity occurs during progression to morula and blastocyst stages (Wright et al. 1996, Betts \& King 1999, Xu \& Yang 2000, Wright et al. 2001). The very high levels of telomerase activity detected in the immature oocytes raises the possibility that telomere elongation occurs 
in these cells during their passage through meiosis. The equally high activity detected at the blastocyst stage of embryonic development suggests that telomere elongation also occurs in the differentiating germ line. The fact that telomerase activity showed high variability among embryonic cells (Wright et al. 2001) indicates that from very early developmental stages some cells may be more proficient than others in telomere elongation. It is still to be established whether the germ line cells are among those with high telomerase activity.

The fact that oocytes at fertilization stage are characterized by low telomerase activity may provide a selection mechanism for cells that have already acquired sufficient telomere length to be fertilized successfully. Consistent with this is the observation by Liu et al. (2002b) that oocytes with short telomeres produced by telomerase-null mice fail fertilization and early cleavage embryonic stages.

\section{The testis}

In contrast with the female germ line, from which oocytes can easily be isolated and analysed individually or in homogenous populations, the testis contains a mixture of cells with various functions, undergoing different processes in an asynchronous manner. For example, spermatogonia undergo mitotic divisions during germ line development, then reach maturation followed by entry into meiosis. Most testis cells are engaged in meiotic divisions (spermatogenesis) to produce haploid gametes, which then undergo a differentiation process (spermiogenesis) characterized by dramatic changes in cell morphology and size to generate mature sperm.

De Lange and colleagues (de Lange et al. 1990) established that sperm telomeres are significantly longer than those of somatic cells; however, telomerase activity has not been detected in mature sperm (Wright et al. 1996, Eisenhauer et al. 1997). Thus, one important aspect of meiotic telomere maintenance that remains to be elucidated is which of the several cell types in the testis are proficient in telomerasemediated telomere elongation, or indeed whether telomerase is the only mechanism of telomere maintenance in the germ line. The major challenge in this direction has been fractionation of synchronous cell populations from testis, which is practically difficult owing to the lack of preferential positioning of various cell types within the seminiferous tubules and also to the similarity in their sizes. Methods for isolation of fractions enriched in specific meiotic stages have been reported, including centrifugal elutriation (Grabske et al. 1975, Meistrich et al. 1977), which can allow enrichment of up to $90 \%$ in pachytene cells, as determined by immunofluorescence staining with markers specific for this stage such as the synaptic protein SYCP1 (Tarsounas et al. 1999).

Eisenhauer and colleagues (Eisenhauer et al. 1997) showed that extracts from fractionated pachytene spermatocytes and round spermatids have high telomerase activity in an in vitro TRAP assay (Kim et al. 1994), while spermatozoa had no detectable activity. However, the composition of these fractions has not been directly determined in these experiments, and the authors acknowledge the possibility of contamination with other types of cells. Other studies have used detection of telomerase expression by in-situ hybridization to establish the timing of telomere elongation in the testis. According to these experiments, expression of the telomerase RNA component peaks in meiosis I spermatocytes and Sertoli cells of adult human testis (Yashima et al. 1998, reviewed in Bekaert et al. 2004), and it is relatively high in all cells of human pre-pubertal testis. However, telomerase expression does not necessarily imply active telomere elongation, therefore, a direct method for in-situ detection of telomerase activity (in-situ TRAP assay) has recently been developed by Tanemura and colleagues (Tanemura et al. 2005). The classical TRAP assay for telomerase activity is based on PCR amplification of telomerase products

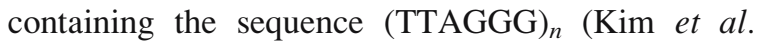
1994). The in situ version of the assay allows detection and quantification of telomere extension in tissue sections, thus directly identifying telomeraseproficient cells. In this assay, telomerase activity peaked in replicating pre-leptotene cells and round spermatids of adult mouse testis, which suggests that telomere elongation occurs before and after meiosis. The in-situ TRAP assay has a great potential for accurate identification of telomerase-proficient cells, particularly if used in combination with molecular markers for various meiotic stages. The downside is that it has not yet been validated in telomerasenegative mice. Tanemura et al. (2005) have also employed telomere restriction fragment analysis (TRF; van Steensel \& de Lange 1997) to demonstrate that telomeric DNA tracts are significantly longer in the testis of sexually mature mice (12 weeks old) 
than in those of pre-pubertal mice (4-weeks old). This is an interesting finding, as it indicates that entry into meiosis triggers telomere lengthening.

\section{Does telomere elongation occur during meiosis?}

The overall image that has emerged from various studies is that telomerase acts in the germ line, and its timing is governed by a sexual dimorphism, consistent with clear differences between meiosis in the two sexes (Figure 1). In oocytes, telomere elongation probably occurs during the first meiotic division, similarly to prophase I spermatocytes. In addition, in the testis, elongation may also occur before cell commitment to meiosis and after completion of meiosis. A reliable method for accurately defining the stage of telomere elongation in the testis or ovary is currently lacking, mainly owing to inefficient methods for isolation of homogeneous populations of cells synchronously undergoing meiosis. This complexity is partly overcome in the ovary where the meiotic arrest facilitates study of certain meiotic stages. The development of methods for in-situ telomerase activity detection in testis holds a promise for future studies, once its specificity is confirmed in the telomerase-negative mice. Molecular markers are nowadays available for accurate identification of individual stages of meiotic progression (e.g. SCP1, SCP3, REC8), which should significantly aid these studies, in conjunction with the widely used morphological criteria implemented by Leblond \& Clermont (1952).

An important mechanism of telomere length regulation in all cells is the assembly of the active form of telomerase at the telomere (Blackburn 2001), which is dependent upon the availability of an open DNA end. Thus, exposure of the telomeric G overhang is a major requirement for successful elongation, and a poorly understood aspect of meiotic telomere biology. Some clues to availability of the $\mathrm{G}$ overhang during meiosis may come from the distribution of the telomeric factor TRF2, crucial for the formation of the t-loop structure with a role in protecting the telomeric G-rich tail (Figure 1). Scherthan and colleagues (Scherthan et al. 2000) have demonstrated that TRF2 is detected at the telomeres of mouse spermatocytes during prophase I. Here we present evidence that TRF2 association
PROPHASE I (PACHYTENE)
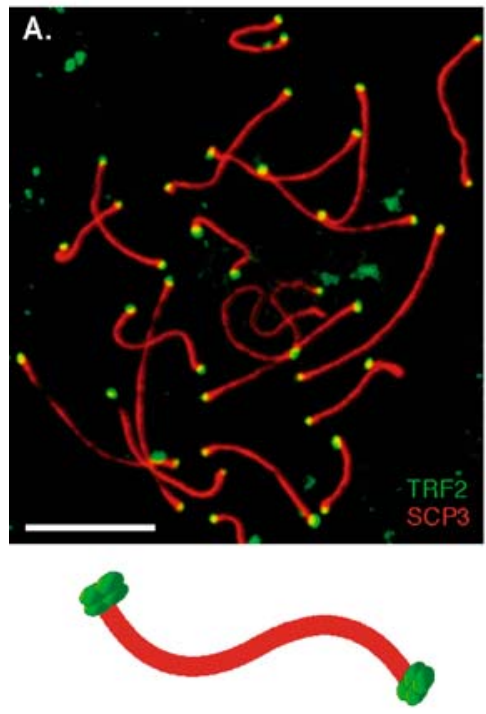

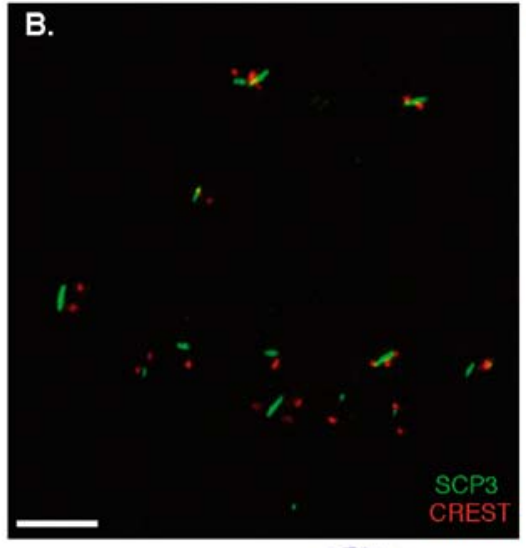

B.

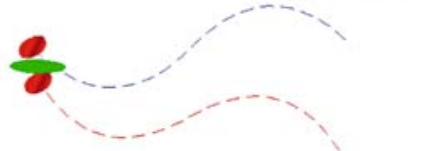

METAPHASE II

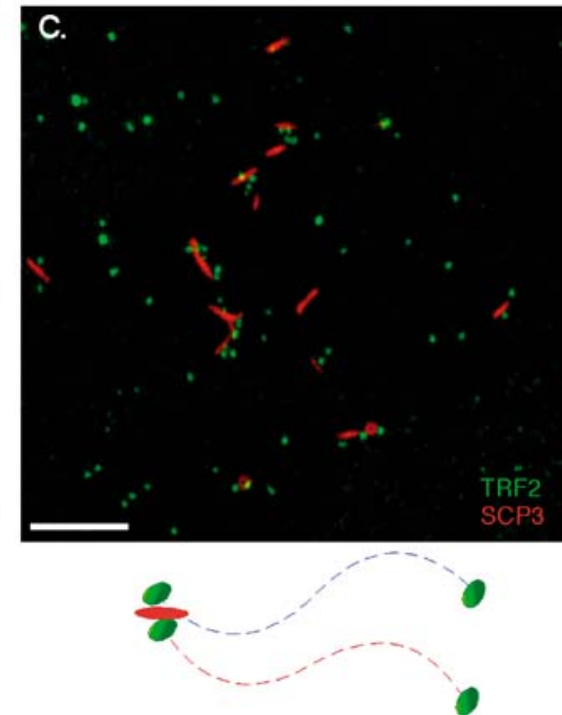

Figure 1. Association of TRF2 with mouse telomeres in meiosis I and meiosis II spermatocytes. (A) TRF2 (green) stains the telomeres of synapsed chromosomes during the pachytene stage of meiosis I. (B) Metaphase II spermatocytes are identified by the characteristic residual SCP3 staining (green) between the separating kinetochores (CREST serum; red). (C) At metaphase II, half of the TRF2-labelled mouse telomeres (green), coinciding with centromeres, associate with residual SCP3 staining (red). Scale bars $=10 \mu \mathrm{m}$. 
with meiotic telomeres can be detected at metaphase II (Figure 1). While the entire length of the meiotic chromosomes can be visualized during first meiotic prophase using antibodies against the SCP3 component of the axial elements (Figure 1A), metaphase II is identifiable by the characteristic residual SCP3 staining between sister centromeres (Figure 1B). As mouse chromosomes are telocentric, half of their telomeres coincide cytologically with the centromeres; therefore, SCP3 is detectable between two sister telomeres (Figure 1C). Presence of TRF2 at the telomeres during both meiotic divisions may suggest that t-loops are assembled and telomeres are inaccessible for elongation throughout meiosis. Alternatively, some TRF2 may remain at telomeres even when the t-loop opens and telomeres are elongated. This is one aspect of telomere maintenance that is not fully understood at the moment. In mitotically diving cells, telomerase extends chromosome ends during $\mathrm{S}$ phase, coincidentally with telomere replication (Marcand et al. 2000). If meiotic cells were to follow the mitotic paradigm, then telomere elongation should occur in the precursor cells undergoing premeiotic replication.

\section{Telomere protection}

A fundamental role of the telomeres is to protect chromosome termini from being sensed by the cell as broken DNA (the capping function; Takai et al. 2003), which can then trigger a DNA damage checkpoint response to elicit cell-cycle arrest that prevents further telomere erosion (diFagagna et al. 2004). Cells can escape this block in cell growth and attempt to repair their uncapped telomeres by engaging the DNA repair machinery, which leads to chromosomal end-to-end fusions. Thus, to preserve chromosome integrity, cells need to discriminate between broken DNA ends and the natural ends of the chromosomes. The mechanism that enables cells to distinguish between the two remains elusive. One possibility is that telomere-binding proteins impose a unique chromatin structure at the telomeres, which prevents their recognition as DSBs. Three proteins that bind telomeric repeats with sequence specificity in vitro have been identified in mammalian cells: TRF1 (Chong et al. 1995) and TRF2 (van Steensel et al. 1998), which bind to double-stranded repetitive telomeric DNA and localize to the telomeres in mammalian cells, and POT1 (Baumann \& Cech 2001), which binds to the $3^{\prime}$ telomeric overhang. These proteins further recruit other factors to the telomere (e.g. TRF1 recruits among others tankyrase and, similarly, TRF2 recruits RAP1 and its modulator, TIN2), ultimately generating a higher-order complex with end-capping and length-control functions (reviewed in Blasco 2005, de Lange 2005).

Recently, homologous recombination, a major pathway for DSB repair within all cells and of special significance for cells undergoing meiotic divisions, has emerged as an important mechanism for telomere protection (Henson et al. 2002). Homologous recombination is thought to remodel telomeric DNA into a structure called the t-loop (Griffith et al. 1999, Figure 2), thus protecting it from DNAdamage sensors. The t-loop may form by invasion of the $3^{\prime}$ telomeric overhang into the duplex DNA of the same telomere, thereby generating a Holliday junction $(\mathrm{HJ})$, the hallmark recombination intermediate (Liu \& West 2004). Telomeric HJs will sequester the invading terminus away from the DNA processing activities and DNA damage sensors present within the cell.

T-loops appear to be evolutionarily conserved structures at chromosome ends (de Lange et al. 2006). The existence of t-loops in meiotic cells has not been formally demonstrated, but they are expected to exist at least in some species from extrapolation of data available for the telomeres of mitotically diving cells. Many aspects related to their assembly, stabilization and dissolution have not yet been elucidated, although the requirement for DNA repair activities has been clearly demonstrated (Verdun \& Karlseder 2006). It has been postulated that some homologous recombination activities, mainly provided by the RAD51 paralog family, may act in protecting or stabilizing the telomeric HJ (Tarsounas \& West 2005). For example, RAD51D is present at meiotic telomeres throughout prophase I, potentially acting in protecting $\mathrm{HJ}$ and telomere integrity (Tarsounas et al. 2004, Figure 3A), consistent with the HJ binding activity demonstrated in vitro for a RAD51Dcontaining complex (Yokoyama et al. 2004). Additionally, the RAD51C/XRCC3 complex associated with $\mathrm{HJ}$ resolution activities in HeLa extracts (Liu et al. 2004b), and with a role in HJ binding and targeting the resolution activity to HJs, is detected in 


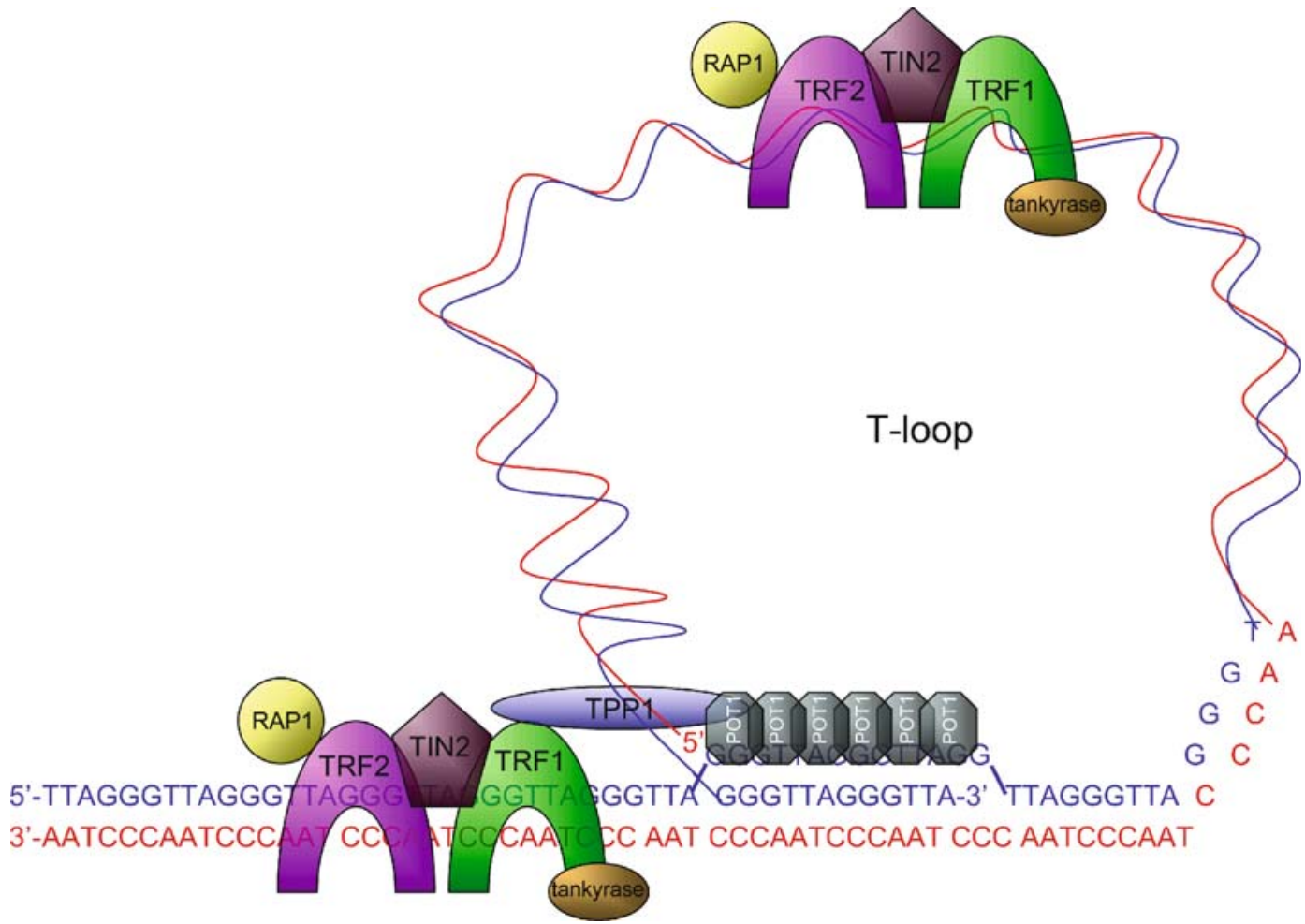

Figure 2. The t-loop telomeric configuration and proteins associated with telomeres in mammalian cells.

association with some meiotic telomeres (Figure 3B). Unprotected telomeres may become vulnerable to endonucleolytic activities, including HJ resolution, which release large fragments of telomeric DNA resulting in telomere shortening (Wang et al. 2004). In addition to RAD51D, the Fanconi anaemia protein FANCD2 has been shown to localize to the telomeres of meiotic chromosomes (Garcia-Higuera et al. 2001). Although cell lines derived from individuals with Fanconi anaemia have been shown to undergo telomere shortening, its function there is not yet understood. Other factors, such as RAD51, RAD52, RAD54, etc. have been demonstrated to act at the mitotic telomeres (Jaco et al. 2003, Verdun \& Karlseder 2006) but their association with meiotic telomeres has not been reported. This could be due to a transient association of these proteins with the meiotic telomeres, or to the limited specificity of the available antibodies (e.g. epitope masking).

TRF2 plays a fundamental role in protecting telomeric DNA overhangs from degradation and preventing end-to-end fusions (van Steensel et al. 1998), possibly by promoting t-loop assembly in vivo
(Griffith et al. 1999, Amiard et al. 2007). In mouse testis, TRF2 is associated with meiotic mouse telomeres throughout prophase I (Scherthan et al. 2000) and is still detectable at meiosis II (Figure 1C), suggesting that the capping function is preserved throughout meiosis. This concept could, in principle, be tested directly by examining meiosis in a TRF2deleted mouse. However, lack of this gene leads to early embryonic lethality (Celli \& de Lange 2005). A floxed TRF2 allele was recently generated (Celli \& de Lange 2005), which should allow conditional deletion in testis. If successful, this would represent an important tool for studying the function of telomere capping during meiosis. So far, the importance of telomere-protective structures in meiosis could only be inferred from studies in yeast. Maddar and colleagues (Maddar et al. 2001) have shown that the end-capping function of the telomere is essential for progression through meiosis in the budding yeast Kluveromyces lactis, and that Rap1, and ortholog of mammalian TRF1 and TRF2, plays an essential role. Similarly, fission yeast Taz1, an ortholog of mammalian TRF2, is essential for spore viability (Cooper et al. 1998). 
A.
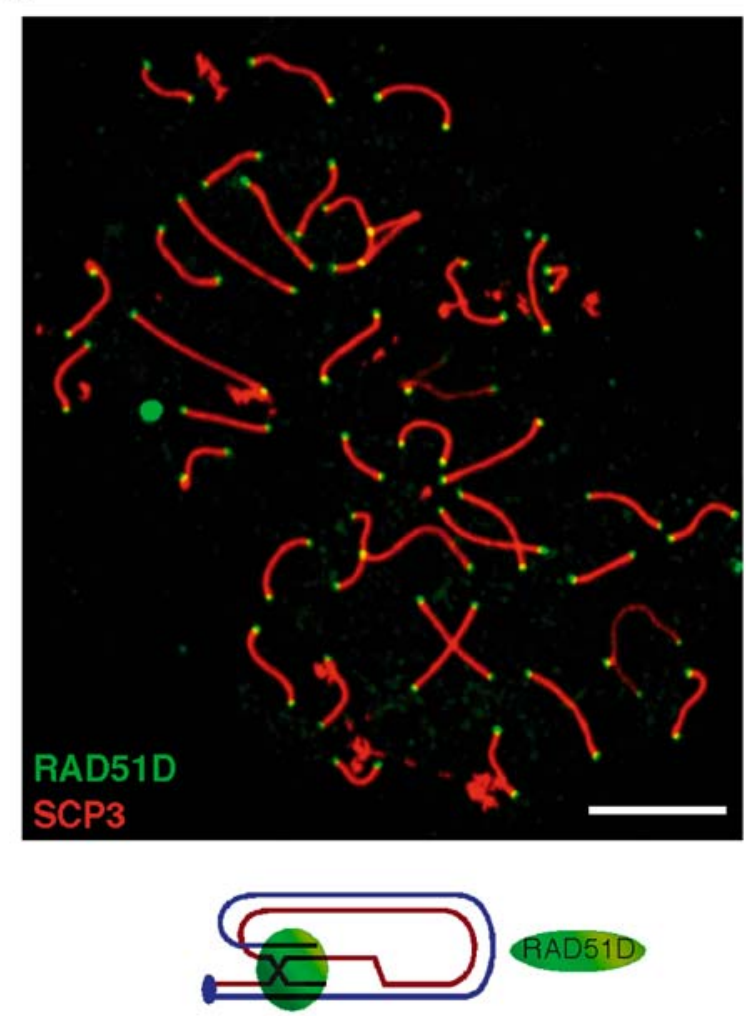

Protection?
B.
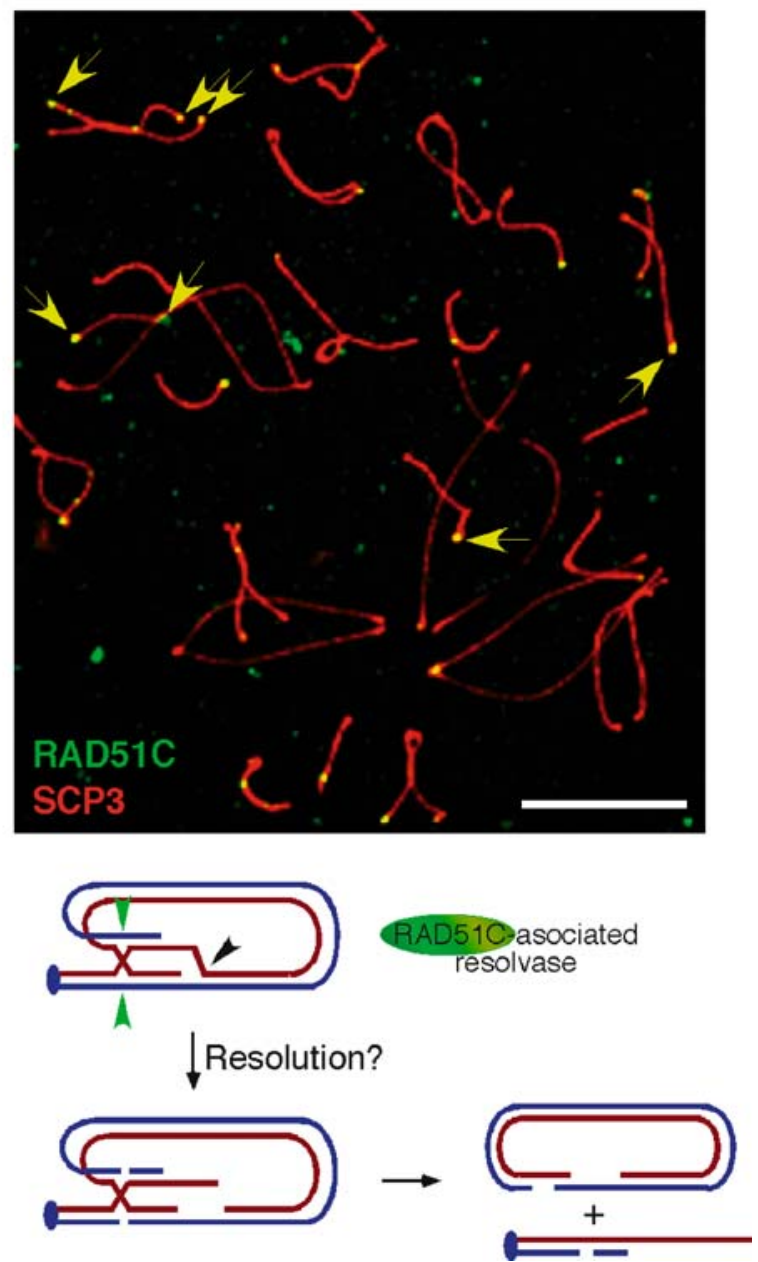

Figure 3. Association of RAD51 paralogs with meiotic telomeres and possible roles in telomere regulation. (A) RAD51D (green) associates with mouse telomeres during meiosis I, where it may stabilize telomeric t-loops. (B) RAD51C (green) binds to a subset of the telomeres (yellow arrows) in mouse spermatocytes, where the RAD51C-associated resolvase activity may be required for telomere processing reactions similar to mitotic TRD. (Reproduced with permission from J Biol Chem (2007) 282: 1973). SCP3, a structural component of meiotic chromosomes, is in red. Scale bars $=10 \mu \mathrm{m}$.

\section{Do telomerase-deficient mice with short telomeres undergo meiosis?}

In 1997, Blasco and colleagues generated the first mice lacking the telomerase RNA gene $\left(T R^{-1-}\right.$ or Terc $^{-1-}$ mice; Blasco et al. 1997). Telomerase activity was completely abolished in these mice, which led to progressive telomere shortening and loss of telomeric capping function with successive generations. While early-generation $\operatorname{Terc}^{-1-}$ mice showed normal fertility in spite of shorter than wildtype telomeres, in the late generations the telomereexhausted chromosomes and associated end-to-end fusions resulted in genomic instability and sterility (Lee et al. 1998, Herrera et al. 1999). Consistent with differences between males and females in meiosis progression, late generation Terc $^{-1-}$ mice exhibited sex-specific meiotic differences: in males, the high rate of apoptosis induces a striking depletion of spermatocytes from the seminiferous tubules (Franco et al. 2002, Liu et al. 2004a), while the female meiosis seems less compromised and is still able to generate oocytes. The tolerance of short telomeres in females can be explained by less stringent checkpoint control of meiotic progression in oocytes, leading to an increased proportion of mature oocytes carrying 
chromosomal aberrations (Hunt \& Hassold 2002). This apparent meiotic competence however, comes at a price, as telomerase-deficient oocytes engage in aberrant fertilization leading to abnormal cleavage of embryos (Liu et al. 2002b).

In late-generation $\operatorname{Terc}^{-1-}$ males, meiotic progression is impaired at the synaptic pachytene stage owing to compromised inter-homolog recombination (Liu et al. 2004a). Short telomeres are inefficient in tethering chromosome ends to the nuclear envelope (Figure 3), which in turn interferes with homologous chromosome alignment and recombination. Similar defects in females impact negatively upon the integrity of meiotic spindles (Liu et al. 2002a), but the rate of elimination in oocytes is far lower, and many reach maturity (Liu et al. 2002b). TRF1 levels in the germ cells of late generation Terc $^{-1-}$ mice are significantly reduced compared to wild type (Franco et al. 2002), consistent with the idea that the amount of TRF1 at each telomere reflects the length of the telomeric tract, similarly to the telomere repeat counting role of Rap1 in budding yeast (Marcand et al. 1997). It still remains to be elucidated whether TRF2, an indicator of the protection status of the telomere, remains associated with short meiotic telomeres in the late-generation telomerase-deficient mice. On the basis of previous results (van Steensel et al. 1998), it is conceivable that TRF2 association with short dysfunctional telomeres is significantly reduced. It is worth emphasizing that no end-to-end fusions have been reported so far in the meiotic cells of the late-generation Terc $^{-1-}$ mice, in contrast with the high incidence of such fusions in somatic cells of the same genotype. This could be due to a yet unknown protection mechanism for very short telomeres upon entry into meiosis, or to the down-regulation of the non-homologous end-joining machinery, commonly required for generating such fusions, during early meiosis (Goedecke et al. 1999).

In mitotically dividing cells, telomere attrition leads to rampant genomic instability, which triggers senescence or apoptosis through activation of the p53 pathway (Maser \& DePinho 2002). Consistent with this, genetic inactivation of p53 partially abrogates several of the phenotypes caused by telomere shortening in TerC $^{-1-}$ mice, including the striking germ cell depletion (Chin et al. 1999). Although the p53 $3^{-1-} \operatorname{Terc}^{-1-}$ mice can be bred for two additional generations compared to the single $\operatorname{Terc}^{-1-}$ mutant mice, they also eventually become sterile. It is conceivable that lack of p53 compromises efficient detection of homologous recombination defects caused by short telomeres in these mice, and allows meiotic progression. However, an in-depth analysis of meiotic progression in these mice might unravel an origin of meiotic failure different from merely short telomeres. Interestingly, erratic telomere elongation has been reported in some seminiferous tubules of the $p 53^{-1-} \operatorname{Terc}^{-1-}$ mice (Chin et al. 1999), which may be due to activation of alternative pathways of telomere lengthening.

\section{ALT-Is it active during meiosis?}

Telomerase provides the major cellular enzymatic activity for direct addition of telomeric DNA repeats to chromosome ends. However, most cells in adult organism lack telomerase activity, which limits their life span. A pathway for telomere elongation alternative to telomerase has been defined in cells lacking telomerase or the ability to activate it, named alternative lengthening of telomeres (ALT), and unlimited proliferation can emerge (Henson et al. 2002). The mechanism of ALT-mediated telomere maintenance is not well understood, but it is thought to engage the homologous recombination machinery in reactions involving recombination between a short telomere and a donor DNA molecule containing telomeric repeats - a longer telomere, an extrachromosomal circle, etc. (reviewed by Henson et al. 2002). These reactions often generate exceedingly long telomeres, which contribute to the heterogeneity in telomere length within the same cell, one of the hallmarks of ALT activation.

Late-generation $\operatorname{Terc}^{-1-}$ mice with telomereexhausted chromosome ends and high apoptotic rate are sterile and often succumb to severe malfunction of various organs (reviewed in Blasco 2005). It is surprising that only very modest activation of telomere-rescue mechanisms has been detected in these mice and this is limited to the germinal centres of the spleen: proliferating splenocytes show abnormally long telomeres upon antigen stimulation (Herrera et al. 2000). More recently, Maria Blasco's group has demonstrated that ALT is activated in Terc-deficient keratinocytes when TRF2 is overexpressed (Blanco et al. 2007). The only indication so far of ALT activation in the germ line comes from the study of late-generation $\operatorname{Terc}^{-1-}$ mice that also lack p53 (Chin et al. 1999). In these mice, most 
seminiferous tubules were depleted of spermatocytes; however, some appeared meiotically active, and their cells had very long telomeres. This suggests that germ cells have the capacity to activate ALT in the absence of telomerase. Although it is not clear where this activation occurred, it can be anticipated that cells at the early stages of meiosis where recombinational DSBs repair occurs could have the ability to engage their already active recombination machinery in elongating their own eroded telomeres.

Lusting and colleagues have recently reported an intriguing role for homologous recombination in telomere maintenance during meiosis in budding yeast (Joseph et al. 2005). The authors postulate the existence of a mechanism for shortening exceedingly long telomeres in meiosis through a mechanism involving inter-chromatid recombination similar to telomere rapid deletion (TRD) in mitotic cells (Lustig 2003). TRD is thought to occur by t-loop resolution and excision of telomeric DNA fragments (Bucholc et al. 2001), and ensures that the length of all telomeres is reset within a narrow distribution at the time cells commit to meiosis. This observation strengthens the possibility that occasional processing of telomeric tract by homologous recombination activities on certain telomeres occurs during meiosis. Whether this reflects active telomere length alteration or just the repair of DSBs accidentally introduced in the telomeric DNA tract is hard to distinguish. Perhaps such occasional processing could explain the sporadic association of homologous recombination proteins with some telomeres but not others on meiotic chromosome spreads (Figure 3B).

\section{Telomere contribution to meiotic chromosome architecture and meiotic spindle integrity}

\section{Nuclear membrane attachment \\ and bouquet formation}

During the early stages of the meiotic prophase I, chromosomes undergo a series of morphological changes including compaction, formation of axial elements that structurally define each homolog and establishment of inter-homolog interactions which facilitate their recombination at DNA level (reviewed in Zickler \& Kleckner 1998). This period also includes the bouquet stage, when telomeres embedded in the inner nuclear membrane move into a polarized configuration and cluster together surrounding the spindle pole body (SPB) in fission yeast or the centrosome in mammals (reviewed in Harper et al. 2004). Attachment of the telomeres to the nuclear envelope and bouquet formation are two distinct aspects of telomere behaviour during meiosis (Zickler \& Kleckner 1998): telomeres remain anchored to the inner surface of the nuclear envelope from leptotene to late pachytene, while the bouquet assembly is a far more transient phenomenon occurring at the leptotene-zygotene transition.

Although bouquet configuration is a universal feature of meiosis, its precise function remains obscure. The severity of its absence for cell viability cannot simply be equated with the subsequent defect in homologous recombination, which has been reported in yeast mutants with defective bouquet formation. It is expected that future studies will unravel some novel essential function for this structure in meiosis. Equally, the molecular basis for telomere clustering remains largely unknown in most eukaryotes. In budding yeast, the telomeric protein Ndj1p seems to be required for both bouquet formation and telomere attachment (Trelles-Sticken et al. 2000). More recently, lack of bouquet formation has also been reported in cells lacking the Set1 histone H3 methyltransferase (Trelles-Sticken et al. 2005). In maize, the paml mutant shows a clustering defect similar to that of $n d j l$; however, nuclear envelope attachment is normal (Golubovskaya et al. 2002). A meiosis-specific protein similar to Ndj1p has not been identified so far in mammalian cells.

The most detailed insights into the molecular mechanism of bouquet formation come from studies in fission yeast. During meiotic prophase in this organism, the nucleus becomes dramatically elongated and engaged in a robust back-and-forth movement between the cell poles. This movement, driven by the SPB and associated telomeres, promotes homolog pairing and keeps homologous chromosomes aligned as they undergo recombination. Mutations in the Taz1 telomere-binding protein reduce telomere clustering and their association with the SPB (Cooper et al. 1998, Nimmo et al. 1998). In addition to the telomeric Taz1, two other factors required for bouquet formation, Bqt1 and Bqt2, have been recently identified in $S$. pombe (Chikashige et al. 2006). Genetic and molecular analyses led to characterization of a complex in which Bqt1 and Bqt2 interact with each other and mediate the 
interaction of the SBP component Sad1 with telomeric factor Rap1, thus allowing bouquet formation. Mammalian SUN-domain protein Sun2, related to S. pombe Sad1, has been shown to localize to the telomeric attachment sites on the nuclear envelope during meiosis (Schmitt et al. 2007). The homologs of Bqt1 and Bqt2 in mammalian cells have not yet been identified. Screening for proteins interacting with RAP1 specifically during meiosis may lead to the identification of these bridging factors and could help define the molecular mechanism of telomere tethering in mammalian cells.

\section{Is the telomeric DNA tract important for attachment and bouquet formation?}

Analysis of chromosome derivatives in various organisms demonstrated that the presence of telomeric DNA repeats is essential for bouquet formation. Human circular chromosomes lacking telomeric sequences do not participate in the bouquet (Voet et al. 2003). Similarly, maize and fission yeast ring chromosomes associate with the SPB as part of the bouquet provided that telomeric sequences are present on these chromosomes (Carlton \& Cande 2002; Sadaie et al. 2003).

It seems that not only the presence but also the length of the telomere repeat is essential for nuclear envelope attachment: short telomeres in late-generation telomerase-deficient mice lack the perinuclear distribution characteristic of mid-prophase I stages (Liu et al. 2004a); however, no effect on bouquet formation has been reported. Thus, the direct link between telomere length and bouquet assembly is still to be established. In these mice, telomere attrition also triggers defects in meiotic homologous recombination and chromosome segregation leading to meiosis failure. This is illustrated by the high rate of apoptosis in the testis and frequent arrest of meiotic progression in females.

Late-generation $\operatorname{Terc}^{-1-}$ mice exhibit short axial elements (Liu et al. 2002a). These, however, may not contribute the meiotic defect in these animals, as correctly assembled axial elements do not appear to be a prerequisite for telomere attachment and clustering in mouse (Liebe et al. 2004). Importantly, correct nuclear envelope attachment plaques and bouquet assembly seem to require components of the cohesin complex in maize and mice (Revenkova et al. 2004, Golubovskaya et al. 2006). Therefore it is possible that cohesin may mediate the interaction between chromosome ends and the SPB/centrosome components during meiosis.

\section{Does homologous recombination control bouquet dissolution?}

An intriguing feature of the bouquet stage is its dissolution, which seems to be dependent upon successful completion of recombination events and therefore could be checkpoint regulated. Correct telomere positioning at the nuclear envelope and into the bouquet configuration facilitates (but does not initiate; Wu \& Burgess 2006) proper alignment of the homologous chromosomes, which is in turn a prerequisite for their recombination (Scherthan 2001, Harper et al. 2004). Telomeres cluster normally in mice lacking various homologous recombination activities, but exit from this stage is delayed (Liebe et al. 2006). This is likely due to activation of an intra-prophase I checkpoint that monitors correct progression of recombination reactions during meiosis. Most likely, this checkpoint response involves ATM (Di Giacomo et al. 2005), a serine-threonine kinase that phosphorylates several DNA repair and checkpoint proteins involved in the repair of the DNA doublestrand breaks that initiate meiotic recombination (Shiloh 2003, Jeggo \& Lobrich 2006).

\section{Spindle integrity}

One of the most striking differences between meiosis in males and females in humans is the rate of meiotic chromosomal errors. Between $10 \%$ and $25 \%$ of fetuses have the wrong chromosome complement (Hassold \& Hunt 2001). The most common errors are trisomies and monosomies, the majority of which are due to non-disjunction at maternal meiosis I, a process in which the spindle integrity and correct kinetochore attachment to the spindle are critical factors. This extraordinarily high rate of erroneous meiosis I in females is thought to be due to less stringent checkpoint control of the first meiotic division, thus allowing alignment and segregation of cells carrying chromosomal abnormalities (Hunt \& Hassold 2002). 
An interesting connection between meiotic progression in oocytes and telomere length has recently been established (Liu et al. 2002a). This work showed that short telomeres in late-generation telomerasenegative mice lead to dramatic defects in chromosome alignment and spindle integrity at metaphase I. However, the vulnerability of metaphase I spindle in these mice may be indirectly affected by telomere length: it is possible that the mechanism underlying misalignment and dysfunctional spindle originates in defects in chromosome pairing and recombination during prophase I, which could have been directly caused by short telomeres. Consistent with a pairing defect, prophase I oocytes show high incidence of unpaired/discontinuous axial elements (Liu et al. 2004a) and detection of bivalents with few or no chiasmata, as determined by lack of MHL1 foci, suggests defects in recombinational DSB repair.

\section{Conclusions}

Although telomeres have been viewed as structures with very specialized function during meiosis, the picture emerging from their molecular characterization suggests that they might share more similarities with telomeres of somatic cells than originally anticipated. Some major questions related to the function of mammalian meiotic telomeres remain to be answered, and one key issue is how telomere capping impacts on meiosis. Availability of conditional mouse models for components of the telomeric complex, allowing specific deletion in ovaries and testis, are expected to provide some insight into how fundamental telomere maintenance mechanisms contribute to meiotic progression. Additionally, the meiotic telomeric complex still awaits a detailed biochemical characterization. Understanding its molecular composition might help elucidate the mysterious function of the telomeric bouquet in meiosis. Alternatively, the more conservative approach of visualizing telomere-associated factors identified in somatic cells at meiotic telomeres would allow formulation of hypotheses on their potential roles during meiosis based on spatiotemporal localization patterns. Ultimately, this type of approach will hopefully enable progress towards a better understanding of how telomeres contribute to human fertility.

\section{Acknowledgements}

We are grateful to Maria Blasco, Frank Uhlmann and Stephane Marcand for critical reading and suggestions on this manuscript. Research in M.T.'s laboratory is funded by Cancer Research UK, Breast Cancer Campaign and The Royal Society.

\section{References}

Amiard S, Doudeau M, Pinte S et al. (2007) A topological mechanism for TRF2-enhanced strand invasion. Nat Struct Mol Biol 14: 147-154.

Baumann P, Cech TR (2001) Pot1, the putative telomere endbinding protein in fission yeast and humans. Science 292: 1171-1175.

Bekaert S, Derradji H, Baatout S (2004) Telomere biology in mammalian germ cells and during development. Dev Biol 274: $15-30$.

Betts DH, King WA (1999) Telomerase activity and telomere detection during early bovine development. Dev Genet 25: 397-403.

Blackburn EH (2001) Switching and signaling at the telomere. Cell 106: 661-673.

Blanco R, Munoz P, Flores JM, Klatt P, Blasco MA (2007) Telomerase abrogation dramatically accelerates TRF2-induced epithelial carcinogenesis. Genes Dev 21: 206-220.

Blasco MA (2005) Telomeres and human disease: ageing, cancer and beyond. Nat Rev Genet 6: 611-622.

Blasco MA, Funk W, Villeponteau B, Greider CW (1995) Functional characterization and developmental regulation of mouse telomerase RNA. Science 269: 1267-1270.

Blasco MA, Lee H-W, Hande MP et al. (1997) Telomere shortening and tumor formation by mouse cells lacking telomerase RNA. Cell 91: 25-34.

Bodnar AG, Ouellette M, Frolkis M et al. (1998) Extension of lifespan by introduction of telomerase into normal human cells. Science 279: 349-352.

Bucholc M, Park Y, Lustig AJ (2001) Intrachromatid excision of telomeric DNA as a mechanism for telomere size control in Saccharomyces cerevisiae. Mol Cell Biol 21: 6559-6573.

Carlton PM, Cande WZ (2002) Telomeres act autonomously in maize to organize the meiotic bouquet from a semipolarized chromosome orientation. J Cell Biol 157: 231-242.

Celli GB, de Lange T (2005) DNA processing is not required for ATM-mediated telomere damage response after TRF2 deletion. Nat Cell Biol 7: 712-718.

Chikashige Y, Tsutsumi C, Yamane M, Okamasa K, Haraguchi T, Hiraoka Y (2006) Meiotic proteins Bqt1 and Bqt2 tether telomeres to form the bouquet arrangement of chromosomes. Cell 125: 59-69.

Chin L, Artandi SE, Shen Q et al. (1999) p53 deficiency rescues the adverse effects of telomere loss and cooperates with telomere dysfunction to accelerate carcinogenesis. Cell 97: $527-538$. 
Chong L, van Steensel B, Broccoli D, Erdjument-Bromage H, Hanish J, Tempst P, de Lange T (1995) A human telomeric protein. Science 270: 1663-1667.

Cooper JP, Watanabe Y, Nurse P (1998) Fission yeast Taz1 protein is required for meiotic telomere clustering and recombination. Nature 392: 828-831.

de Lange T (2002) Protection of mammalian telomeres. Oncogene 21: $532-540$.

de Lange $T$ (2005) Shelterin: the protein complex that shapes and safeguards human telomeres. Genes Dev 19: 2100-2110.

de Lange T, Shiue L, Myers RM et al. (1990) Structure and variability of human chromosome ends. Mol Cell Biol 10: $518-527$.

de Lange T, Lundblad V, Blackburn E (2006) Telomeres, 2nd edn. New York: CSH Laboratory Press.

diFagagna FD, Teo SH, Jackson SP (2004) Functional links between telomeres and proteins of the DNA-damage response. Genes Dev 18: 1781-1799.

Di Giacomo M, Barchi M, Baudat F, Edelmann W, Keeney S, Jasin M (2005) Distinct DNA-damage-dependent and -independent responses drive the loss of oocytes in recombination-defective mouse mutants. Proc Natl Acad Sci USA 102: 737-742.

Eisenhauer KM, Gerstein RM, Chiu C-P, Conti M, Hsueh AJW (1997) Telomerase activity in female and male rat germ cells undergoing meiosis and in early embryos. Biol Reprod 56: 1120-1125.

Flores JM, Cayuela ML, Blasco MA (2005) Effects of telomerase and telomere length on epidermal stem cell behavior. Science 309: 1253-1256.

Franco S, Alsheimer M, Herrera E, Benavente R, Blasco MA (2002) Mammalian meiotic telomeres: composition and ultrastructure in telomerase-deficient mice. Eur J Cell Biol 81: 335-340.

Garcia-Higuera I, Taniguchi T, Ganesan S et al. (2001) Interaction of the Fanconi anemia proteins and BRCA1 in a common pathway. Mol Cell 7: 249-262.

Goedecke W, Eijpe M, Offenberg HH, van Aalderen M, Heyting C (1999) Mre11 and Ku70 interact in somatic cells, but are differentially expressed in early meiosis. Nat Genet 23: 194-198.

Golubovskaya IN, Harper LC, Pawlowski WP, Schichnes D, Cande WZ (2002) The pam1 gene is required for meiotic bouquet formation and efficient homologous synapsis in maize (Zea mays L.). Genetics 162: 1979-1993.

Golubovskaya IN, Hamant, O, Timofejeva L et al. (2006) Alleles of afd1 dissect REC8 functions during meiotic prophase I. J Cell Sci 119: 3306-3315.

Grabske RJ, Lake S, Gledhill BL, Meistrich ML (1975) Centrifugal elutriation: separation of spermatogenic cells on the basis of sedimentation velocity. J Cell Physiol 86: 177-189.

Greider CW, Blackburn EH (1985) Identification of a specific telomere terminal transferase activity in Tetrahymena extracts. Cell 43: 405-413.

Griffith JD, Comeau L, Rosenfield S et al. (1999) Mammalian telomeres end in a large duplex loop. Cell 97: 503-514.

Harley CB, Futcher AB, Greider CW (1990) Telomeres shorten during ageing of human fibroblasts. Nature 345: 458-460.

Harper L, Golubovskaya I, Zacheus Cande W (2004) A bouquet of chromosomes. J Cell Sci 117: 4025-4032.
Hassold T, Hunt P (2001) To err (meiotically) is human: the genesis of human aneuploidy. Nat Rev Genet 2: 280-291.

Hemann MT, Strong MA, Hao LY, Greider CW (2001) The shortest telomere, not average telomere length, is critical for cell viability and chromosome stability. Cell 107: 67-77.

Henson JD, Neumann AA, Yeager TR, Reddel RR (2002) Alternative lengthening of telomeres in mammalian cells. Oncogene 21: 598-610.

Herrera E, Samper E, Martın-Caballero J, Flores JM, Lee H-W, Blasco MA (1999) Disease states associated with telomerase deficiency appear earlier in mice with short telomeres. EMBO J 18: 2950-2960.

Herrera E, Carlos Martinez A, Blasco, MA (2000) Impaired germinal center reaction in mice with short telomeres. $E M B O$ J 19: 472-481.

Hiyama E, Hiyama K (2002) Clinical utility of telomerase in cancer. Oncogene 21: 643-649.

Hunt PA, Hassold TJ (2002) Sex matters in meiosis. Science 296: 2181-2183.

Jaco I, Munoz P, Goytisolo F et al. (2003) Role of mammalian Rad54 in telomere length maintenance. Mol Cell Biol 16: 5572-5580.

Jeggo PA, Lobrich M (2006) Contribution of DNA repair and cell cycle checkpoint arrest to the maintenance of genomic stability. DNA Repair (Amst) 5: 1192-1198.

Joseph I, Jia D, Lustig AJ (2005) Ndj1p-dependent epigenetic resetting of telomere size in yeast meiosis. Curr Biol 15: 231-237.

Kim NW, Piatyszek MA, Prowse KR et al. (1994) Specific association of human telomerase activity with immortal cells and cancer. Science 266: 2011-2015.

Leblond CP, Clermont Y (1952) Definition of the stages of the cycle of the seminiferous epithelium in the rat. Ann NY Acad Sci 55: 548-573.

Lee HW, Blasco MA, Gottlieb GJ, Horner JWn, Greider CW, DePinho RA (1998) Essential role of mouse telomerase in highly proliferative organs. Nature 392: 569-574.

Liebe B, Alsheimer M, Hoog C, Benavente R, Scherthan H (2004) Telomere attachment, meiotic chromosome condensation, pairing, and bouquet stage duration are modified in spermatocytes lacking axial elements. Mol Biol Cell 15(2): 827-837.

Liebe B, Petukhova G, Barchi M et al. (2006) Mutations that affect meiosis in male mice influence the dynamics of the midpreleptotene and bouquet stages. Exp Cell Res 312: 3768-3781.

Liu L, Blasco MA, Keefe DL (2002a) Requirement of functional telomeres for metaphase chromosome alignments and integrity of meiotic spindles. EMBO Rep 3: 230-234.

Liu L, Blasco MA, Trimarchi JR, Keefe DL (2002b) An essential role for functional telomeres in mouse germ cells during fertilization and early development. Dev Biol 249: 74-84.

Liu Y, West SC (2004) Happy Hollidays: 40th anniversary of the Holliday junction. Nat Rev Mol Cell Biol 5: 937-944.

Liu L, Franco S, Spyropoulos B, Moens PB, Blasco MA, Keefe DL (2004a) Irregular telomeres impair meiotic synapsis and recombination in mice. Proc Natl Acad Sci USA 101: 6496-6501.

Liu Y, Masson JY, Shah R, O'Regan P, West SC (2004b) RAD51C is required for Holliday junction processing in mammalian cells. Science 303: 243-246.

Lustig AJ (2003) Clues to catastrophic telomere loss in mammals from yeast telomere rapid deletion. Nat Rev Genet 4: 916-923. 
Maddar H, Ratzkovsky N, Krauskopf A (2001) Role for telomere cap structure in meiosis. Mol Biol Cell 12: 3191-3203.

Marcand S, Gilson E, Shore D (1997) A protein-counting mechanism for telomere length regulation in yeast. Science 275: 986-990.

Marcand S, Brevet V, Mann C, Gilson E (2000) Cell cycle restriction of telomere elongation. Curr Biol 10: 487-490.

Maser RS, DePinho RA (2002) Connecting chromosomes, crisis, and cancer. Science 297: 565-569.

McEachern MJ, Krauskopf A, Blackburn EH (2000) Telomeres and their control. Annu Rev Genet 34: 331-358.

Meistrich ML, Grdina DJ, Meyn RE, Barlogie B (1977) Separation of cells from mouse solid tumors by centrifugal elutriation. Cancer Res 37: 4291-4296.

Nimmo ER, Pidoux AL, Perry PE, Allshire RC (1998) Defective meiosis in telomere-silencing mutants of Schizosaccharomyces pombe. Nature 392: 825-828.

Revenkova E, Eijpe M, Heyting C et al. (2004) Cohesin SMC1 $\beta$ is required for meiotic chromosome dynamics, sister chromatid cohesion and DNA recombination. Nat Cell Biol 6: 555-562.

Sadaie M, Naito T, Ishikawa F (2003) Stable inheritance of telomere chromatin structure and function in the absence of telomeric repeats. Genes Dev 17: 2271-2282.

Samper E, Goytisolo FA, Menissier-de Murcia J, Gonzales-Suarez E, Cigudosa JC, de Murcia G, Blasco MA (2001) Normal telomere length and chromosomal end capping in poly(ADPribose) polymerase-deficient mice and primary cells despite increased chromosomal instability. J Cell Biol 154: 49-60.

Scherthan H (2001) A bouquet makes ends meet. Nat Rev Mol Cell Biol 2: 621-627.

Scherthan H, Jerratsch M, Li B et al. (2000) Mammalian meiotic telomeres: protein composition and redistribution in relation to nuclear pores. Mol Biol Cell 11: 4189-4203.

Schmitt J, Benavente R, Hodzic D, Hoog C, Stewart CL, Alsheimer M (2007) Transmembrane protein Sun2 is involved in tethering mammalian meiotic telomeres to the nuclear envelope. Proc Natl Acad Sci USA 104: 7426-7431.

Shiloh Y (2003) ATM and related protein kinases: safeguarding genome integrity. Nat Rev Cancer 3: 155-168.

Takai H, Smogorzewska A, de Lange T (2003) DNA damage foci at dysfunctional telomeres. Curr Biol 13: 1549-1556.

Tanemura K, Ogura A, Cheong C et al. (2005) Dynamic rearrangement of telomeres during spermatogenesis in mice. Dev Biol 281: 196-207.

Tarsounas M, West SC (2005) Recombination at mammalian telomeres: an alternative mechanism for telomere protection and elongation. Cell Cycle 4: 672-674.

Tarsounas M, Pearlman RE, Moens PB (1999) Meiotic activation of rat pachytene spermatocytes with okadaic acid: the behaviour of synaptonemal complex components SYN1/SCP1 and COR1/ SCP3. J Cell Sci 112: 423-434.

Tarsounas M, Munoz P, Claas A et al. (2004) Telomere maintenance requires the RAD51D recombination/repair protein. Cell 117: 337-347.
Trelles-Sticken E, Dresser ME, Scherthan H (2000) Meiotic telomere protein $\mathrm{Ndj} 1 \mathrm{p}$ is required for meiosis-specific telomere distribution, bouquet formation and efficient homologue pairing. J Cell Biol 151: 95-106.

Trelles-Sticken E, Bonfils S, Sollier J, Géli V, Scherthan H, de La Roche Saint-André C (2005) Set1- and Clb5-deficiencies disclose the differential regulation of centromere and telomere dynamics in Saccharomyces cerevisiae meiosis. J Cell Sci 118: 4985-4994.

van Steensel B, de Lange T (1997) Control of telomere length by the human telomeric protein TRF1. Nature 385: 740-743.

van Steensel B, Smogorzewska A, de Lange T (1998) TRF2 protects human telomeres from end-to-end fusions. Cell 92: 401-413.

Verdun RE, Karlseder J (2006) The DNA damage machinery and homologous recombination pathway act consecutively to protect human telomeres. Cell 127: 709-720.

Voet T, Liebe B, Labaere C, Marynen P, Scherthan H (2003) Telomere-independent homologue pairing and checkpoint escape of accessory ring chromosomes in male mouse meiosis. $J$ Cell Biol 162: 795-807.

Wahl GM, Carr AM (2001) The evolution of diverse biological responses to DNA damage: insights from yeast and p53. Nat Cell Biol 12: 277-286.

Wang RC, Smogorzewska A, de Lange T (2004) Homologous recombination generates T-loop-sized deletions at human telomeres. Cell 119: 355-368.

Watson JD (1972) Origin of concatemeric T7 DNA. Nat New Biol 239: 197-201.

Wright DL, Jones EL, Mayer JF, Oehninger S, Gibbons WE, Lanzendorf SE (2001) Characterization of telomerase activity in the human oocyte and preimplantation embryo. Mol Hum Reprod 7: 947-955.

Wright WE, Piatyszek MA, Rainey WE, Byrd W, Shay JW (1996) Telomerase activity in human germline and embryonic tissues and cells. Dev Genet 18: 173-179.

Wu H-Y, Burgess SM (2006) Ndj1, a telomere-associated protein, promotes meiotic recombination in budding yeast. Mol Cell Biol 26: $3683-3694$.

Xu J, Yang X (2000) Telomerase activity in bovine embryos during early development. Biol Reprod 63: 1124-1128.

Yashima K, Maitra A, Barton Rogers B et al. (1998) Expression of the RNA component of telomerase during human development and differentiation. Cell Growth Differ 9: 805-813.

Yokoyama H, Sarai N, Kagawa W et al. (2004) Preferential binding to branched DNA strands and strand-annealing activity of the human Rad51B, Rad51C, Rad51D and Xrcc2 protein complex. Nucleic Acids Res 32: 2556-2565.

Zickler D, Kleckner N (1998) The leptotene-zygotene transition of meiosis. Аnnu Rev Genet 32: 619-697.

Zijlmans JM, Martens UM, Poon SS et al. (1997) Telomeres in the mouse have large inter-chromosomal variations in the number of T2AG3 repeats. Proc Natl Acad Sci USA 94: 7423-7428. 